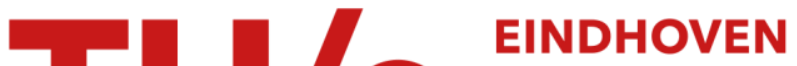 UNIVERSITY OF TECHNOLOGY
}

\section{Multiple nonergodic disordered states in Laponite suspensions : a phase diagram}

Citation for published version (APA):

Jabbari-Farouji, S., Tanaka, H., Wegdam, G. H., \& Bonn, D. (2008). Multiple nonergodic disordered states in Laponite suspensions : a phase diagram. Physical Review E - Statistical, Nonlinear, and Soft Matter Physics, 78(6), 061405-1/10. [061405]. https://doi.org/10.1103/PhysRevE.78.061405

DOI:

10.1103/PhysRevE.78.061405

Document status and date:

Published: 01/01/2008

\section{Document Version:}

Publisher's PDF, also known as Version of Record (includes final page, issue and volume numbers)

\section{Please check the document version of this publication:}

- A submitted manuscript is the version of the article upon submission and before peer-review. There can be important differences between the submitted version and the official published version of record. People interested in the research are advised to contact the author for the final version of the publication, or visit the $\mathrm{DOI}$ to the publisher's website.

- The final author version and the galley proof are versions of the publication after peer review.

- The final published version features the final layout of the paper including the volume, issue and page numbers.

Link to publication

\section{General rights}

Copyright and moral rights for the publications made accessible in the public portal are retained by the authors and/or other copyright owners and it is a condition of accessing publications that users recognise and abide by the legal requirements associated with these rights.

- Users may download and print one copy of any publication from the public portal for the purpose of private study or research.

- You may not further distribute the material or use it for any profit-making activity or commercial gain

- You may freely distribute the URL identifying the publication in the public portal.

If the publication is distributed under the terms of Article 25fa of the Dutch Copyright Act, indicated by the "Taverne" license above, please follow below link for the End User Agreement:

www.tue.nl/taverne

Take down policy

If you believe that this document breaches copyright please contact us at:

openaccess@tue.nl

providing details and we will investigate your claim. 


\title{
Multiple nonergodic disordered states in Laponite suspensions: A phase diagram
}

\author{
S. Jabbari-Farouji, ${ }^{1,2}$ Hajime Tanaka, ${ }^{3}$ G. H. Wegdam, ${ }^{1}$ and Daniel Bonn ${ }^{1,4}$ \\ ${ }^{1}$ Van der Waals-Zeeman Institute, University of Amsterdam, 1018XE Amsterdam, The Netherlands \\ ${ }^{2}$ Theoretical Physics and Polymer Group, Department of Applied Physics, Technische Universiteit Eindhoven, 5600MB Eindhoven, \\ The Netherlands \\ ${ }^{3}$ Institute of Industrial Science, University of Tokyo, Meguro-ku, Tokyo 153-8505, Japan \\ ${ }^{4}$ Laboratoire de Physique Statistique de l'ENS, 75231 Paris Cedex 05, France
}

(Received 21 April 2008; revised manuscript received 22 September 2008; published 23 December 2008)

\begin{abstract}
We study the time evolution of different Laponite suspensions from a low-viscosity ergodic state to a viscoelastic nonergodic state over a wide range of volume fractions and salt contents. We find that the evolution of nonergodicity parameter (Debye-Waller factor) splits into two branches for all the samples, which correspond to two distinct dynamically arrested states. At moderately high salt concentrations, on the other hand, a third nonergodic state appears that is different from the above two nonergodic states. Measurement of the conductivity of Laponite solutions in pure water shows that the contribution of counterions in the ionic strength is considerable and their role should be taken into account in interpretations of aging dynamics and the phase diagram. Based on these data and available data in the literature, we propose a (nonequilibrium) phase diagram for Laponite suspensions.
\end{abstract}

DOI: 10.1103/PhysRevE.78.061405

PACS number(s): 61.43.Fs, 81.40.Cd, 64.70.-p, 64.90.+b

\section{INTRODUCTION}

Understanding the phase behavior of clay suspensions is of important technical and scientific interest. Characterization of different ordered and disordered phases formed by clays is of direct importance for various industrial applications such as soil mechanics and, for instance, the control of viscoelastic properties of materials with clay additives. Of more fundamental importance is the study of the underlying mechanism behind gelation and glass formation that are both observed in clays. This potentially provides us with a deeper understanding of dynamically arrested states of matter. Clay suspensions can typically be modelled as charged anisotropic particles such as disks immersed in an electrolyte, thus interacting via excluded volume, long-range electrostatic repulsions and weak (van der Waals) attractions. The phase diagram of anisotropic charged colloids such as clays and understanding the aggregation, gelation, and glass formation appearing in such systems is a matter of considerable debate [1-8]. The specific clay system we study here, Laponite (a synthetic clay [9]) has been the subject of intensive study over the past decade or more.

Laponite consists of crystalline platelets with a thickness of $1 \mathrm{~nm}$ and an average diameter about $30 \mathrm{~nm}$ and a bulk density of $2.6 \mathrm{~g} / \mathrm{cm}^{3}$ [9]. Each Laponite particle is a threelayer silicate composed of a central octahedrally coordinated magnesium-oxygen-hydroxide sandwiched between two tetrahedrally coordinated silica-oxygen sheets. Isomorphic substitutions of the divalent magnesium atoms in the central layer by monovalent Lithium atoms lead to the formation of negative charges within the lattice, which is balanced by the sodium ions located at the surface. When Laponite is dispersed in water or any polar liquid, the polar molecules penetrate between interleaf regions, dissolving the interleaf cations and separating platelet surfaces by hydration and electrostatic forces. Thus in the final suspension the Laponite surface has negative charge on the order a few thousand electron charge (in water), while its edges (depending on the $p \mathrm{H})$ may have a small localized positive or negative charge generated by desorption or absorption of hydroxyl group where the crystal structure terminates.

For a range of Laponite concentrations and salt contents, the dispersion of Laponite in water is followed by spontaneous evolution from an liquidlike state to a nonergodic solidlike state. This process is called aging, meaning that the physical observables of the system such as diffusion of the particles and dispersion viscosity evolve with time.

The aging dynamics during the fluid-solid transition in Laponite suspensions has been independently studied by many groups $[2-4,7,10-28]$ using light scattering and rheology techniques. Perhaps the earliest studies on the phase behavior come from Mourchid et al. [2] and Kroon et al. [11]. Mourchid et al. attempted to characterize the phase diagram of Laponite suspensions based on rheological measurements [2]. They varied both particle concentration and ionic strength and by performing oscillatory shear measurements on samples 1 week after their preparation they defined a solgel transition line where the zero frequency elastic shear modulus increases remarkably. Their phase diagram gives a general overview, but we have come to realize that their method is flawed as the measurements were done after some arbitrary waiting time $t_{w}$ and viscoelastic properties depend on $t_{w}$.

On the other hand, Kroon et al. [11] studied the aging of Laponite using dynamic light scattering (DLS) experiments. They measured a range of sample concentrations between 2.2 and $3.5 \mathrm{wt} \%$ and found that all the samples show a similar aging behavior and evolve from an initially ergodic state to a nonergodic state around a certain time (ergodicitybreaking point detected by changes in the moments of scattered intensity distribution) that decreases exponentially with increasing concentration. For a 3 wt. \% sample of Laponite, they reported the growth of the nonergodicity parameter (fraction of frozen-in density fluctuations) from almost zero to approximately 0.8 at the late stage of aging. Bonn et al. 
[3] suggested that aging in samples with no added salt is due to strong electrostatic repulsions, leading to the formation of a low volume fraction Wigner glass. They determined the liquid-glass transition volume fraction as a function of ionic strength, assuming that the effective volume per particle can be estimated as $\pi R^{2} l_{D}$ (here $R \approx 15 \mathrm{~nm}$ is the particle radius and $l_{D}$ is the Debye length) by considering the Debye length as the particle thickness. If this exceeds the volume available per particle, which is estimated as $\pi R^{2} h / \phi(h=1 \mathrm{~nm}$ the actual thickness $\phi$ is the volume fraction) no free volume is available and thus a glassy state may emerge. The volume fraction for the liquid-glass transition is given by $\phi_{\mathrm{eff}} \approx 0.5$ and thus varies as $\phi_{l g} \sim h / l_{D} \propto I^{1 / 2}$ where $I$ is the ionic strength. Following the suggestion of Bonn et al. [15], Levitz et al. [13], by deionizing Laponite indeed found the evidence for a Wigner glass at very low ionic strengths consistent with the proposal of Bonn et al.

Nicolai and Cocard $[18,19]$ have studied the aging at low concentrations of Laponite with added salt in the ergodic regime of aging. They observed that the scattered intensity increased with the waiting time. At the late stages of aging, when the scattered intensity has become constant, the structure factor $S(q)$ shows a power-law $q$-dependence characteristic of fractal structures for $S(q)$. In a later paper the same group proposed a revised state diagram for Laponite suspensions based on visual observations and waiting timedependent static light scattering experiments [4]. According to this study, the transition from liquid to "solid" (defined as the state that does not flow when the tube containing the sample is tilted) occurs at much lower concentrations than what was proposed in the phase diagram of Mourchid et al. [2]. From their observations they argue that the origin of aging for all their measured samples with salt and without salt $(C<2$ wt. \%) is gelation rather than glass formation [4].

The systematic study of Ruzicka et al. [22,23] was the first one to show that nonergodic states of Laponite can exist at very low concentrations $(C \approx 0.3 \mathrm{wt}$. \%) of Laponite, in the region which was proposed to be a sol according to the phase diagram of Mourchid et al. [2]. Using DLS, Ruzicka et al. [22,23] systematically studied the aging dynamics of both low and high concentrations and also varied the salt concentration. They showed that the intensity correlation functions at low and high concentrations evolve in a distinctly different manner and two distinct master curves have been identified. They suggested that aging at low concentrations proceeds by formation of a Wigner glass made of Laponite clusters, while at higher concentrations a glass is formed whose basic unit is a single Laponite particle [22]. They show that this result is not affected by the presence of salt and one still finds two distinct routes of aging [23]. However, based on their recent small angle x-ray scattering measurements, Ruzicka et al. [8] conclude rather that the arrested state at low concentrations should be called a gel and at high concentrations an attractive glass.

The study of Ruzicka et al. is the most comprehensive and systematic one up to now. However, their experiments considered only the ergodic regime of aging and they do not present any results in the interesting range of concentrations $1.5<C<2.2$, precisely the range between dilute and concentrated systems. In a recent paper [7], for samples in the range
$0.1<C<3.6$ we showed that in the nonergodic regime the nonergodicity parameter (Debye-Waller factor) also falls onto either of two distinct master curves. This is consistent with the classification obtained based on evolution of dynamic structure factor in the ergodic regime of aging [22].The evolution of nonergodicity parameter provides us with some valuable information about the nature of nonergodic states. Using this information altogether with other data such as short-time diffusion and structure factor at low $q$ limit, we identified the two observed distinct states as gels and glasses [7]. Furthermore we showed that for a range of intermediate colloid concentrations $1.1<C<2.4$, the transition to nonergodicity can occur in either direction (gel or glass). The distinction between glass and gel was mainly made on the basis of (i) the difference in the dynamic structure factor, notably the absence (gel) or presence (glass) of cage-rattling motion; (ii) the difference in static structure factor: $S(q)$ showed power-law behavior as a function of $q$ and a clear increase in time for the gel state. Both are consistent with formation of some sort of structure (cluster or network) in the gel, and are absent in the glassy state.

It may be evident from these observations that the nature of nonequilibrium phases formed by Laponite suspensions remains ambiguous. Both gelation $[2,4]$ and glass formation $[3,16,22,28]$ have been proposed to account for the aging process. Gelation and the glass transition have important similarities. Both are ergodic to nonergodic transitions that are kinetic, rather than thermodynamic in origin, and distinguishing between these two types of nonergodic states experimentally is a longstanding controversy [2-6]. Here we will show that at least part of the confusion about glassy or gel-like behavior of Laponite suspensions finds its origin in that each group has only studied a specific range of concentrations or salt content. Furthermore, most of the studies have been performed in the ergodic regime of aging. Other serious discrepancies between the results of different groups arise from the fact that some of the measurements for determining phase diagram ignored the aging features of Laponite and were done after some arbitrary waiting time $t_{w}[2,13]$. Another important confusion regarding the phase diagram in the literature is the neglect of the ionic strength that originates from the release of sodium ions from the platelets when Laponite is dissolved in water [3]. The ionic strength resulting from the release of sodium ions can be estimated from conductivity measurements. We will show in the following that the ionic strength of Laponite suspensions in pure water is relatively large and cannot be ignored for determining the "effective particle size" from adding the Debye length to the size. Furthermore, different groups have used different grades (XLG and RD) and batches of Laponite which also can change the results slightly. Therefore, this has lead to apparent contradictions for the results reported by different groups, that we attempt to clear up in the present paper.

Here we have extended our previous study [7] to Laponite samples with added salt, since an important part of the discussion about the phase diagram is related to samples to which salt is added to screen the electrostatic repulsion between the Laponite particles. We report here extensive light scattering measurements during the evolution from an initially ergodic liquidlike state to a nonergodic solidlike state 

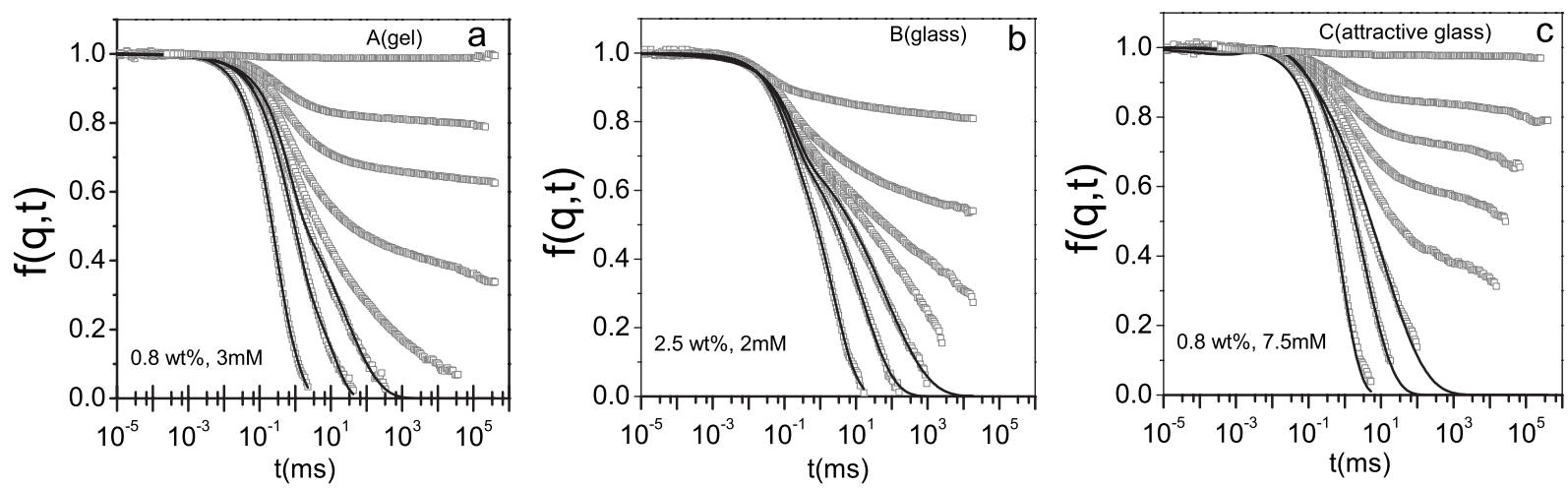

FIG. 1. Evolution of intermediate scattering function $f(q, t)$ for three Laponite samples with salt at scattering angle of $90^{\circ}$. The symbols present the measured correlation functions at increasing waiting times (from left to right) that are $\left(t_{w}=0.075,5.7,7.3,8.8,9.7,11.9,15,19\right.$, and 500 days) for 0.8 wt. $\%, 3 \mathrm{mM}\left(t_{w}=11,104,153,205,255,366\right.$, and $\left.2854 \mathrm{~min}\right)$; for $2.5 \mathrm{wt} . \%, 2 \mathrm{mM}$ salt; and $\left(t_{w}=9,44,66,90,119\right.$, 164,311 , and $3900 \mathrm{~min}$ ) for $0.8 \mathrm{wt} . \%, 7.5 \mathrm{mM}$ salt. In all panels, the lines, on the curves that decay to zero (ergodic stage), show the fits with $f(q, t)=A \exp \left(-t / \tau_{1}\right)+(1-A) \exp \left[-\left(t / \tau_{2}\right)^{\beta}\right]$.

on these samples. We show that in the presence of moderate amounts of added salt, in addition to the two distinct nonergodic states $(A$ and $B)$ of Laponite, reported before [7,22], even a third option $(C)$ appears to exist for the system.

We discuss the nature of the three distinct dynamically arrested states, $A-C$, we identify these, with the help also of data of other groups in the existing literature, in different regions of the phase diagram. This allows us to propose a unifying phase diagram for the nonequilibrium states of Laponite.

\section{EXPERIMENT}

We study charged colloidal disks of Laponite XLG, with an average radius of $15 \mathrm{~nm}$ and $1 \mathrm{~nm}$ thickness. Laponite can absorb water, increasing its weight up to $20 \%$. Therefore, we first dried it in an oven at $100{ }^{\circ} \mathrm{C}$ for 1 week and subsequently stored it in a desiccator.

We prepare a number of Laponite samples with different concentrations and salt contents. Laponite solutions without added salt are prepared in ultrapure millipore water $\left(18.2 \mathrm{M} \Omega \mathrm{cm}^{-1}\right)$ and are stirred vigorously by a magnetic stirrer for $1.5 \mathrm{~h}$ to make sure that the Laponite particles are fully dispersed. The dispersions are filtered using Millipore Millex AA $0.8 \mu \mathrm{m}$ filter units to obtain a reproducible initial state [3]. This instant defines the zero of waiting time, $t_{w}$ $=0$.

The samples with added salt $(\mathrm{NaCl}$, from Sigma) are prepared by diluting the Laponite suspensions in pure water with a more concentrated salt solution [18]. For instance, a sample of 0.8 wt. $\%, 6 \mathrm{mM} \mathrm{NaCl}$ is prepared by mixing equal volumes of $1.6 \mathrm{wt}$ \% Laponite solution in pure water with the same volume of a $12 \mathrm{mM}$ salt solution.

A standard dynamic light scattering setup $(\lambda=632.8 \mathrm{~nm})$ with a coherence factor close to $1 \quad(\approx 0.98)$ measures the time-averaged intensity correlation functions [Eq. (1)] in VV mode, i.e., when polarization of incident light and scattered light are both perpendicular (vertical) relative to the scattering plane,

$$
g_{\mathrm{t}}(q, t)=\frac{\langle I(q, t) I(q, 0)\rangle_{t}}{\langle I(q, 0)\rangle_{t}^{2}}
$$

where $\langle\cdots\rangle_{t}$ stands for the time average. In the ergodic regime of aging this is related to the electric field correlation function, i.e., intermediate scattering function $f(q, t)$ through the Siegert relation $g_{t}(q, t)=1+\beta|f(q, t)|^{2}$, where $\beta$ is an experimental factor close to one [29]. For all the aging samples, there is a certain waiting time after which the timeaveraged correlation functions are no longer equal to their ensemble-averaged values, i.e., they change from one position to another in the sample. This defines the ergodicitybreaking point $t_{e b}$. This point is experimentally determined as the waiting time for which the time-averaged normalized second moment $\left\langle I(t)^{2}\right\rangle_{t} /\langle I(t)\rangle_{t}^{2}$ of the scattered intensity $I(t)$ is not equal to 1 anymore, in other words the measured normalized correlation function $g_{t}$ does not decay from 1 to 0 anymore [11].

For waiting times $t_{w}>t_{e b}$, we calculate the ensembleaveraged electric field correlation function, i.e., intermediate scattering function $f(q, t)$ from the time-averaged intensity correlation function $g_{t}(q, t)$ and ensemble-averaged intensity $I_{E}$ measured by rotating the sample at different heights [30],

$$
f(q, t)=1+\left(I_{t} / I_{E}\right)\left\{\left[g_{t}(q, t)-g_{t}(q, 0)+1\right]^{1 / 2}-1\right\} .
$$

The measurements are performed at scattering wave vector $q=\frac{4 \pi n}{\lambda} \sin \left(\frac{\theta}{2}\right)$, where $\theta=90^{\circ}$ is the scattering angle. The correlation functions are measured at a rate depending on the speed of aging of different Laponite suspensions.

\section{RESULTS}

Measuring the intensity correlations of scattered light from a large number of aging Laponite suspensions, one always observes two regimes of aging in the evolution of the intensity correlation functions. In the first regime the system is ergodic, whereas the second regime corresponds to a nonergodic (arrested) state. The crossover from the former to the latter occurs at ergodicity-breaking time $t_{e b}$ corresponding to the time that part of degrees of freedom are frozen in on the 
time scale of measurements. Figure 1 shows the evolution of ensemble-averaged intermediate scattering functions $f(q, t)$ for three different samples. In all the cases, the correlation functions evolve from an ergodic state to a nonergodic state as the system ages. One can also observe that this generic behavior is not affected by the presence of salt.

The intermediate scattering functions in the ergodic regime can be fitted with the functional form $f(q, t)$ $=A \exp \left(-t / \tau_{1}\right)+(1-A) \exp \left[-\left(t / \tau_{2}\right)^{\beta}\right]$, in which $\tau_{1}$ and $\tau_{2}$ represent the fast and slow relaxation times, respectively $[14,22]$. In the nonergodic regime, the aging rate of the system can be quantified by measuring the time evolution of the nonergodicity parameter $f\left(q, \infty, t_{w}\right)=\lim _{t \rightarrow \infty} f\left(q, t, t_{w}\right)$ [30]. In the absence of salt we have already shown that the evolution of nonergodicity parameter in a range of samples with different Laponite concentrations collapses onto two distinct master curves when plotted as a function of reduced waiting time $\left(t_{w} / t_{e b}-1\right)$ [Fig. 2(a) ]. These branches were interpreted [7] as belonging to a colloidal gel and colloidal glass state based on different aging behavior in other measured quantities. Here we refer to the branches for low and high concentrations as $A$ and $B$, respectively. The observed differences between $A$ and $B$ in the absence of salt can be summarized as follows.

(i) The static structure $S(q)$ of $B$ changes very little with waiting time, while that of $A$ evolves dramatically. This is due to formation of networklike structure or aggregation. This difference manifests itself in the evolution of scattered intensity with waiting time, which grows for $A$ but is nearly constant for $B$ [7].

(ii) The short-time diffusion of particles in $B$ decreases only slightly while it drops significantly in $A$ during the ergodic to nonergodic transition [7] [see also Fig. 4(a)].

(iii) The slow relaxation time of $B$ grows exponentially with waiting time, while that of $A$ grows faster than exponentially $[7,22]$.

(iv) The distribution of relaxation times is different between $A$ and $B$ : $A$ has a broad distribution, whereas $B$ has a double-peaked broad distribution of relaxation times [7].

(v) The difference between $A$ and $B$ is perhaps best reflected in their $q$ dependence of structure factor at low $q$ values and for late waiting times. While the structure factor of $B$ is flat at low $q$ values, $S(q)$ of $A$ is $q$ dependent, indicating that a structure has been formed [7].

(vi) The nonergodicity parameter for $A$ grows at a faster rate than for $B$. While the nonergodicity parameter for $A$ asymptotically reaches one, the nonergodicity parameter for $B$ reaches an approximate value of 0.85 for late waiting times, indicating that there is still some freedom for the particles to move. This is suggestive of the "cage rattling" picture of glassy dynamics, also in agreement with the distributions of relaxation times [7].

As explained in [7], in view of the above measurements, it is tempting to identify $A$ with a gel, and $B$ with a glassy phase. This will be discussed in detail below; we now first consider the effect of added salt.

It is clear that adding salt $(\mathrm{NaCl})$ to a given concentration of Laponite accelerates the aging. Figure 3(a) shows the ergodicity-breaking time for the three samples as a function of salt concentration. The effect is tremendous: By adding a

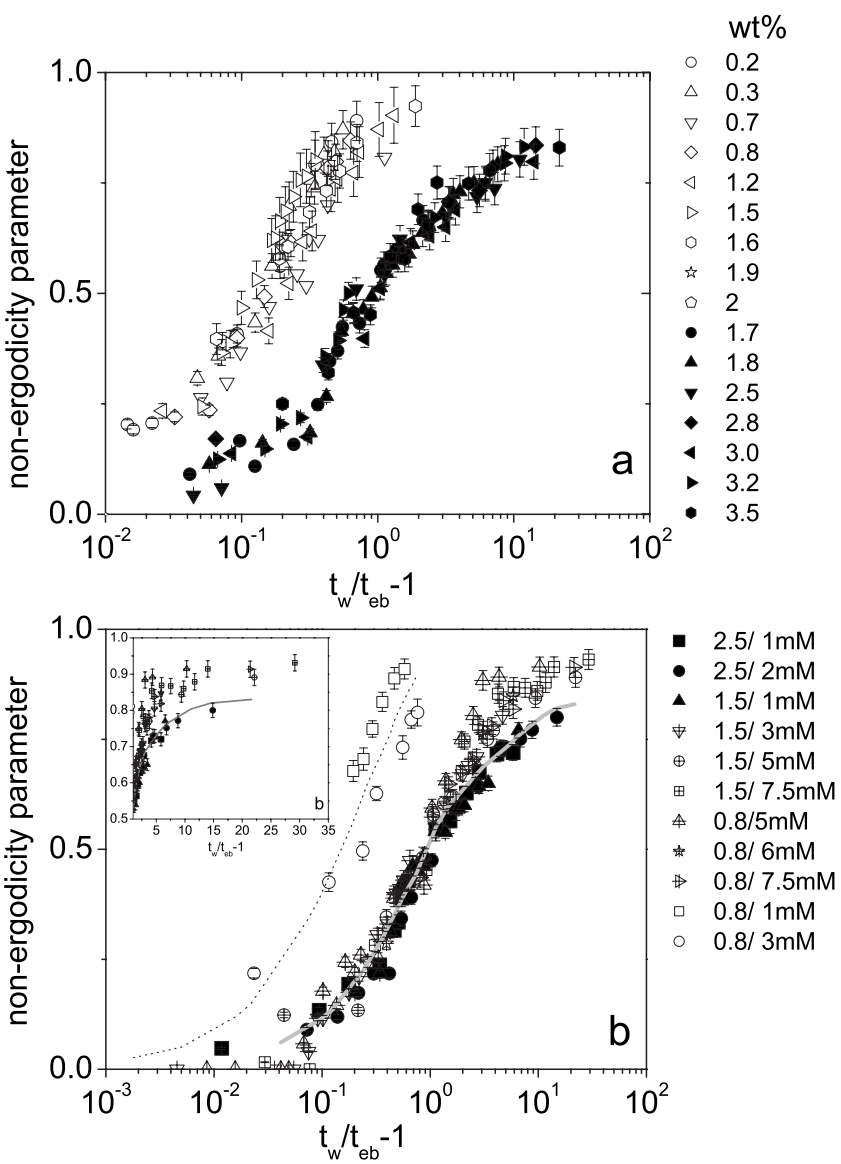

FIG. 2. The evolution of the nonergodicity parameter $f\left(q, \infty, t_{w}\right)$ versus reduced waiting time $t_{w} / t_{e b}-1$ for different Laponite samples (a) without salt and (b) with salt. The colloid concentrations and salt contents are shown in the legend. The samples can be divided into two groups according to the evolution of nonergodicity parameters. In part (b) the dashed and solid lines correspond to the aging process of group $A$ and $B$, respectively, which are obtained from smoothed averaging over the data of Laponite in pure water. As can be seen, for most of the samples with salt (group $C$ ) the nonergodicity parameter deviates from the glass line at long waiting times. The inset of panel (b) shows the difference in nonergodicity parameter between the glass (line, averaged over a large number of experiments) and the attractive glass (data points).

few $\mathrm{mM}$ of salt, $t_{e b}$ can decrease by 4 orders of magnitude, with a roughly exponential dependence of the ergodicity breaking time on salt concentration. We can see the change of the slope in Fig. 3(a) for $0.8 \mathrm{wt} \%$ around $5 \mathrm{mM}$ salt concentration, which reflects the crossover from $A$ to $C$.

Furthermore, looking at the structure factor at the late stages of aging (when the scattering intensity has been stabilized, $\left.50 t_{e b}<t_{w}<100 t_{e b}\right)$ for different salt contents, we find that with increasing salt the intensity increases and the wave-vector dependence of $I(q)$ becomes more pronounced [Fig. 3(b)]. The observed change of the structure factor with an increase in salt is plausible. The more salt we add, the more we suppress the repulsive interactions, therefore the attractive interactions play a more dominant role, leading to formation of denser clusters and a more heterogenous structure. This result is consistent with the results of Refs. $[4,20]$ 

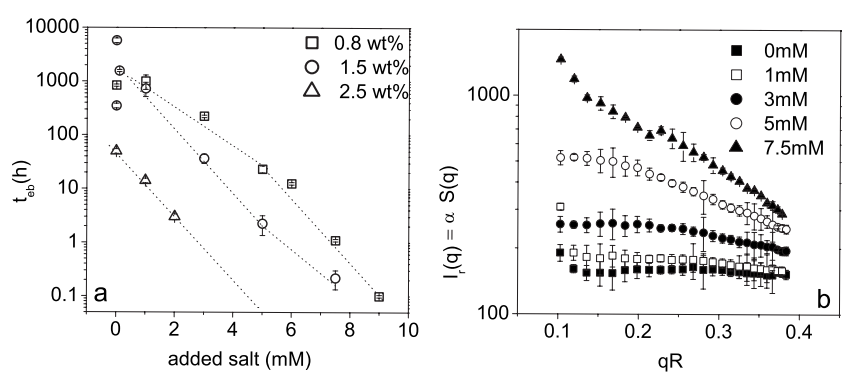

FIG. 3. (a) The ergodicity-breaking time $t_{e b}$ as a function of the salt content for a few Laponite concentrations. $t_{e b}$ decreases with adding salt. The dotted lines here are just guidelines for the eyes. (b) The scattered intensity relative to the toluene intensity $I_{r}$ as a function of dimensionless scattering vector $q R$ for different amounts of added salt $(\mathrm{NaCl})$ at a concentration of 0.8 wt. \%, as shown in the legends. These data are taken a long time after the samples have become fully nonergodic.

in which the dependence of final structure on salt content has been studied for several Laponite concentrations $(0.1,1$, and 1.5 wt. $\%)$.

Figure 2(b) shows that in spite of the accelerated aging, the evolution of nonergodicity parameter $f(q, \infty)$, versus scaled waiting time $t_{w} / t_{e b}$ still splits into two branches similar to those observed before for samples without salt [7].

However, looking carefully at Fig. 2(b), it is evident that some of the samples with salt deviate from the glass $(B)$ master curve (obtained from the data without salt) for longer waiting times. These samples seem to evolve faster than the glass for long waiting times $t_{w}>3 t_{e b}$ and the nonergodicity parameter reaches values higher than for the glass, indicating a blocking of rescaled particle motion. Measurements performed on these samples at very long waiting times show that the nonergodicity parameter of these samples asymptotically reach the value 1 . Hence, it turns out that in the presence of salt, the story is more complicated than the scenario sketched without salt [7]. If we look, for instance, at the scattered intensity as a function of time (Fig. 4), we find that for most of the samples with salt the scattered intensity increases and concomitantly their diffusion coefficient decreases with waiting time. Both increase of intensity and decrease of short-time diffusion are in principle indicative of the building up of structure, and thus suggest that a gel forms [7], $A=$ gel. Comparing, however, with the master curves for the nonergodicity parameter, we find that the high-salt concentration samples (Laponite 0.8 wt. \% with 5 and $7.5 \mathrm{mM}$ ) behave more like $B$, whereas the low-salt concentration samples (Laponite $0.8 \mathrm{wt} \%$ with 1 and $3 \mathrm{mM}$ ) are on the master curve of $A$. All of the 1.5 wt. \% Laponite samples should be glassy also, according to the nonergodicity parameter criterion; however at least for the $1.5 \mathrm{wt} \%, 7.5 \mathrm{mM}$ sample also a clear increase in intensity is observed.

Plotting the slow relaxation time $\tau_{2}$ normalized to its value at $t_{w} \approx 0$ as a function of scaled waiting time $t_{w} / t_{e b}$, we find that $\tau_{2} / \tau_{0}$ for all the samples, with or without salt, splits into two branches (Fig. 5). We can see that for most of the samples belonging to the $B$ branch of nonergodicity parameter the slow relaxation time grows exponentially while for most of the samples belonging to the $A$ branch $\tau_{2}$ grows
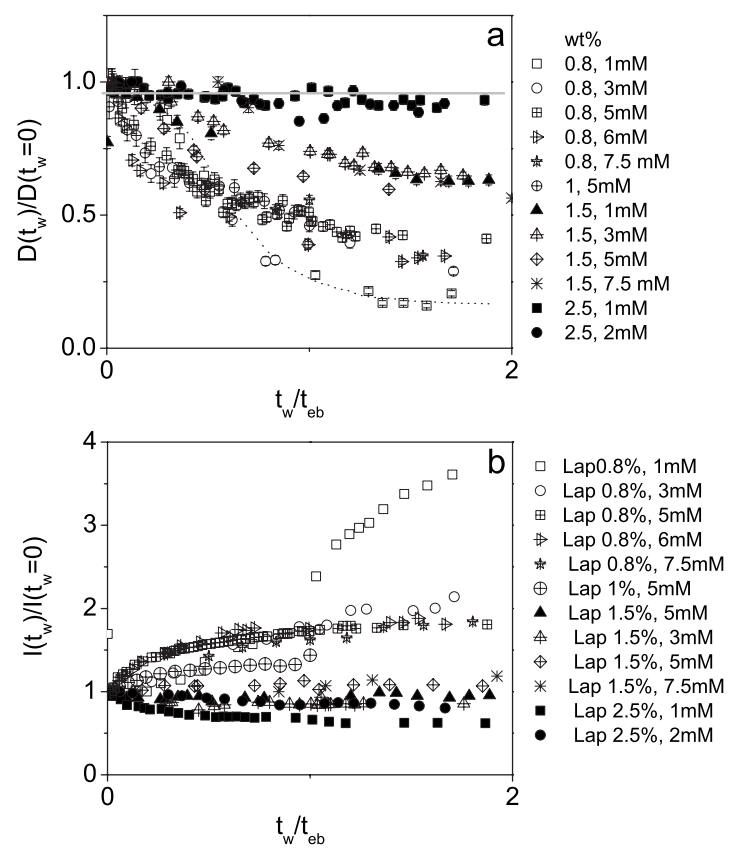

FIG. 4. (a) The evolution of short-time translational diffusion normalized to its initial value $\left(t_{w} \approx 0\right)$ as a function of $t_{w} / t_{e b}$. The solid and dashed lines show the $B$ and $A$ line, respectively, obtained from smoothed averaging over the data of Laponite in pure water. (b) Scattering intensity at scattering angle $90^{\circ}$ as a function reduced waiting time. So as to focus on the effect of aging, we have normalized the intensity to its value at the beginning of aging.

faster than exponentially, similar to what observed for samples without salt [7,22].

Comparing between different quantities, an inconsistency appears, which is always the same one. Looking at the nonergodicity parameter, all the samples at moderate salt always behave like $B$. However, some of their other measured quantities such as scattered intensity, short-time diffusion, and slow relaxation time, consistently behave as if the sample were $A$. This situation is indeed quite different from the one without salt as described in [7]. We see no hesitations of the samples between two states in the sense that a sample that starts evolving in one direction ends up in the other one.

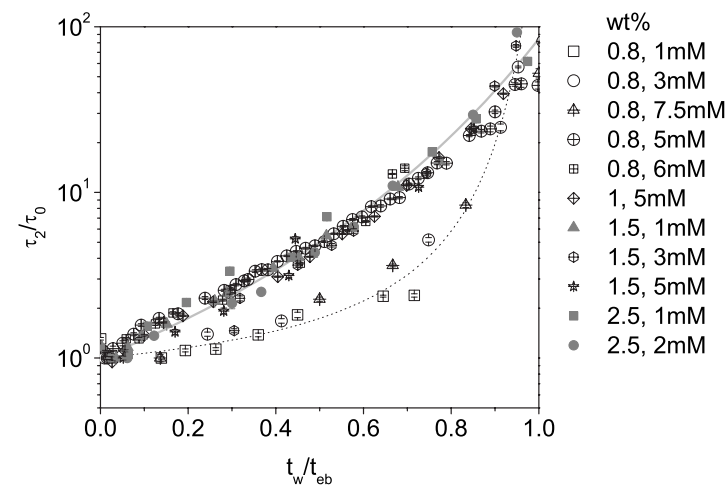

FIG. 5. The evolution of slow relaxation time as a function of the scaled waiting time $t_{w} / t_{e b}$. The solid and dashed lines show the $B$ and $A$ line, respectively, obtained from smoothed averaging over the data of Laponite in pure water. 

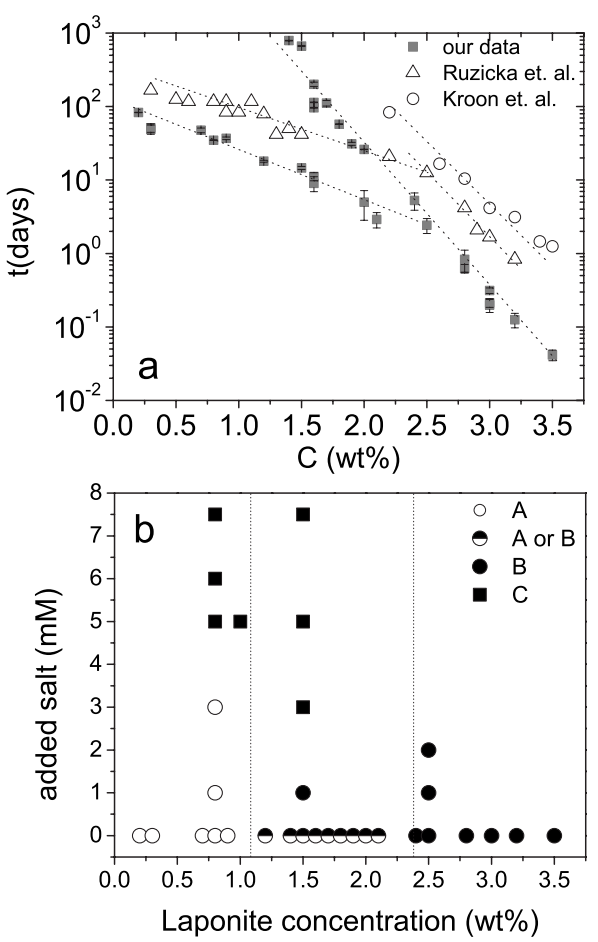

FIG. 6. (a) The ergodicity-breaking time $t_{e b}$ extracted from our data and Kroon et al. data [11] and $t_{w}^{\infty} \propto t_{e b}$ extracted from Ruzicka et al. work [22] as a function of concentration of Laponite in pure water. (b) Our proposed phase diagram based on light scattering data for nonequilibrium states of Laponite.

Rather, all individually measured quantities consistently show an evolution in one direction. The data therefore suggest that although the sample has some definite characteristics of $B$, the other characteristics are those of $A$.

Hence, to summarize, the addition of salt introduces patterns in the aging behavior in the sense that there are samples which share some of the properties of $A$ and some of the features of $B$. We call this set of samples $C$. With this classification, with added salt the samples Laponite 0.8 wt. $\%$ with 5 and $7.5 \mathrm{mM}$ salt and Laponite $1.5 \mathrm{wt}$ \% with 3, 5, and $7.5 \mathrm{mM}$ belong to the group $C$. The samples $2.5 \mathrm{wt} \%$ with 1 and $2 \mathrm{mM}$ salt behave in all respects identically to the group $B$ samples: They are glassy. Also samples $0.8 \mathrm{wt} \%, 1$ and $3 \mathrm{mM}$ behave exactly like group $A$ samples, and therefore are gels.

\section{PHASE DIAGRAM FOR NONEQUILIBRIUM STATES OF Laponite}

As we discussed in detail above, different groups have studied the phase behavior of Laponite suspensions $[2,11,19,22]$, without a consensus emerging. Here, we would like to show that one can get a consistent picture putting all different pieces of information from different groups, despite the fact that each group has used different batches of Laponite and sometimes different grades (Laponite XLG and RD). To demonstrate this point, we have plotted in Fig. 6(a) the ergodicity-breaking time obtained from our measurements [7], from Kroon et al. experiments [11] as well as $t_{w}^{\infty}$

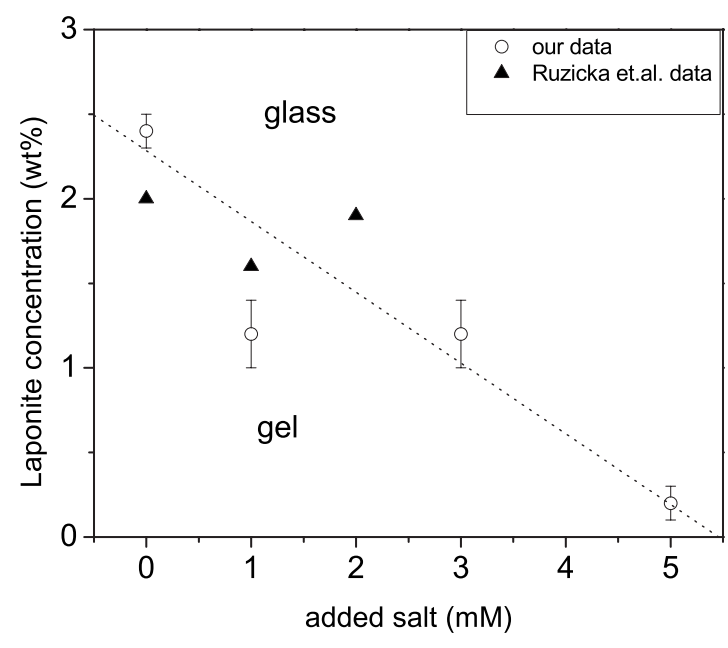

FIG. 7. The approximate $A$ to $B$ transition line obtained from our data and data in Ref. [23].

defined by Ruzicka et al. [22]. Ruzicka et al. obtained $t_{w}^{\infty}$ from fitting the waiting-time-dependent mean relaxation time $\tau_{m}$ with the general form $\tau_{m}\left(t_{w}\right)=\tau_{0} \exp \left(B \frac{t_{w}}{t_{w}^{\infty}-t_{w}}\right)$. Following the proposal of Ruzicka et al. [22], we fitted the mean relaxation time from our data to the above form in order to determine $t_{w}^{\infty}$. Comparing $t_{w}^{\infty}$ obtained from our fits with $t_{e b}$ obtained directly from the experiments, it turned out that for lower concentrations $t_{e b} \approx t_{w}^{\infty}$ and for higher concentrations $t_{e b} \approx 0.6 t_{w}^{\infty}$. Therefore, $t_{w}^{\infty}$ can also be interpreted as a characteristic time for the transition from fluidlike to solidlike state. Figure 6(a) clearly shows that despite the difference in aging speed which is due to different batches of Laponite, the concentration dependence of ergodicity-breaking time found by the different groups is very similar. The two main reasons for the observed differences are most likely the presence of additional water in the experiments of Kroon et al. [11] (the Laponite was not dried), and the difference in salt impurities between the very pure XLG (our experiments) and RD (Kroon et al. [11] and Ruzicka et al. [22]). A new phase diagram for nonequilibrium states of Laponite based on our characterization is shown in Fig. 6(b). Our data suggest the existence of three distinct nonergodic states A-C as demonstrated above. The Ruzicka et al. data also reveal the existence of two different arrested states (called IG1 and IG2 in their paper) for Laponite samples in pure water and at low salt content. Indeed the phase IG1 of Ruzicka et al. corresponds to what we call $A$ and their phase IG2 to $B$. The consistency between their and our data becomes even clearer if we plot the concentration at which the transition from $A$ to $B$ occurs as a function of added salt, as depicted in Fig. 7. As can be seen there is a fair agreement for location of $A$ to $B$ transition line obtained from our data and the Ruzicka et al. data.

Note that in the phase diagram of Fig. 6(b) the coordinate ( $y$ axis) is the amount of added salt, while the interparticle interactions between particles are controlled by the total number of ions in the solution, i.e., the counterions released from the surface of the Laponite particles plus the ions from the added salt. To get an idea about the number density of counterions from Laponite, we have measured the conduc- 

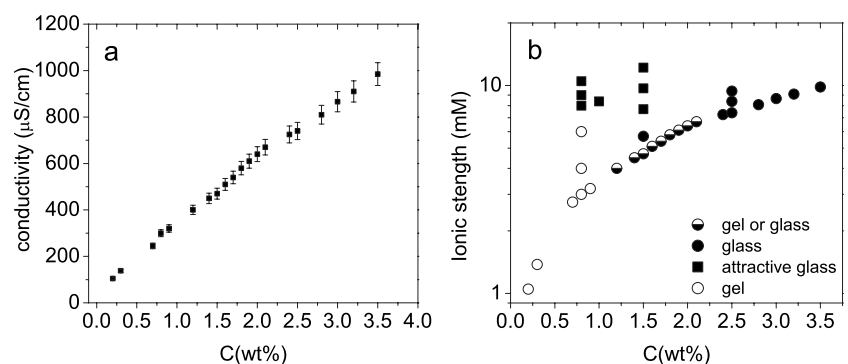

FIG. 8. (a) The conductivity of Laponite suspensions in pure water as a function of concentration measured at early stages of aging, i.e., $t_{w} \approx 0$. (b) The phase diagram with modified salt axis for taking into account the ionic strength resulting from counterions in the solution.

tivity of Laponite solutions. Figure 8(a) shows the conductivity of Laponite solutions in pure water as a function of concentration measured at early stages of aging. We also measured the conductivity values for later stages of aging, before the samples become solidlike. We observed only very small changes, at most an increase in the conductivity of $5 \%$ as a function of waiting time was found. The measured conductivity is mainly due to the $\mathrm{Na}^{+}$counterions released from surface of Laponite particles. The contribution of $\mathrm{OH}^{-}$ions released from the edges of Laponite particles is relatively small. Neglecting this contribution, the number density of $\mathrm{Na}^{+}$ions $n_{\mathrm{Na}}$ can be obtained from $n_{\mathrm{Na}}=\sigma_{\mathrm{Na}} / \mu_{\mathrm{Na}} e$, where $\mu_{\mathrm{Na}}$ is the mobility of $\mathrm{Na}^{+}$ions $\left(\mu_{\mathrm{Na}}=5.19\right.$ $\times 10^{-8} \mathrm{~m}^{2} \mathrm{~s}^{-1} \mathrm{~V}^{-1}$ [31]) and $e$ is the electron charge. The number density of Laponite particles can be estimated as $n_{L}=\Phi_{m} / \rho_{L} v_{L}$ where $\Phi_{m}$ is the mass fraction of Laponite particles, $\rho_{L}$ and $v_{L}$ are the density and volume of an individual Laponite particle. $\left(\rho_{L}=2.6 \mathrm{~g} / \mathrm{cm}^{3}\right.$ is used here and $v_{L}$ is calculated assuming a disk radius of $15 \mathrm{~nm}$ and thickness of $1 \mathrm{~nm}$.) This allows us to plot a phase diagram [see Fig. 8(b)] with $y$ axis being the total ionic strength $I=1 / 2 \sum_{i} n_{i} Z_{i}^{2}$, in which $n_{i}$ is the number density of ion species $i$ and $Z_{i}$ is the charge of the ion species $i$. The abscissa is the Laponite concentration. As can be seen the ionic strength resulting from counterions is considerable and cannot be ignored.

Therefore the total ionic strength in Laponite solutions in pure water is much higher than the $0.1 \mathrm{mM}$ salt below which the Wigner glass was predicted [3] and observed by Levitz et al. [2]. Taking into account the contribution of counterions resolves the confusion about the absence or presence of Wigner glass in Laponite suspensions in pure water. Levitz et al. prepared Laponite solution of extremely low ionic strengths by deionizing Laponite suspensions and immersing until the desired ionic strength. As a result they could observe a low volume fraction solidlike state. Measuring the structure factor with ultrasmall angle $\mathrm{x}$-ray scattering, they correctly identified this phase as a Wigner glass driven mainly by long-range electrostatic repulsions.

\section{DISCUSSION}

To summarize, we have shown that Laponite suspensions can form different types of nonergodic states $(\mathrm{A}-\mathrm{C})$ upon

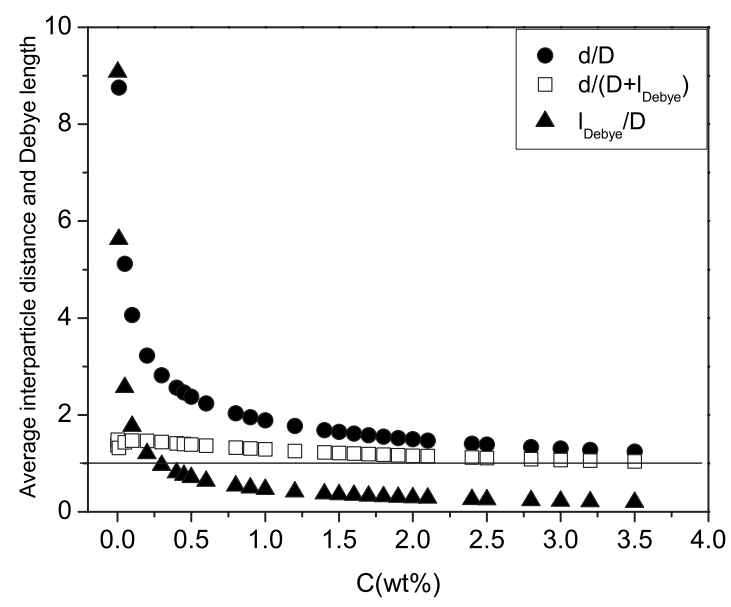

FIG. 9. The ratio of average interparticle distance $d$ to particle size $D=30 \mathrm{~nm}$ and particle size plus Debye length $D+l_{\text {Debye }}$ as a function of concentration for Laponite suspensions in pure water.

changing concentration and salt content. We have shown that the evolution of the nonergodicity parameter (Debye-Waller factor) falls into distinct branches for all Laponite and salt concentrations.

Now we come to the most important question of what is the nature of states $A-C$. For samples without salt, there are the two branches, $A$ and $B$. The key difference is in the evolution of the static structure factor and translational diffusion coefficient with waiting time. The static structure factor and short-time translational diffusion of $B$ are independent of waiting time, while the same quantities are strong functions of the waiting time for $A$. In addition, the slow relaxation time in $B$ grows exponentially with waiting time, whereas it grows faster than exponentially in $A$.

In group $B$ samples (high Laponite concentrations, no or little added salt), the spatial structure is homogenous. This group shows similar aging patterns in the late stage as seen in hard sphere glasses [30] and simulations of Laponite in the glassy state [32]. For instance, even in the latest stages of aging, particles maintain their freedom of rattling in the cages formed by their neighboring particle, as evidenced by waiting time independent short-time diffusion, and a nonergodicity parameter less than 1 , which never exceeds 0.85 even at the latest stage of aging.

We suggest to call this group a repulsive glass in the same sense as the glass formed at high concentrations of hard spheres. Note that although both attractive and repulsive interactions are present in all ranges of Laponite and salt concentrations, we believe that attractions do not play a dominant role at these relatively high concentrations, as is evidenced by the homogenous structure of these suspensions. This analogy becomes clearer if we plot the ratio of average interparticle distance between Laponite particles $d$ to particle size $D=2 R=30 \mathrm{~nm}$ versus concentration (see Fig. 9). $d$ is estimated as $\left(\pi R^{2} h / \phi\right)^{1 / 3}$, where $\phi$ is the volume fraction of Laponite particles. Figure 9 clearly demonstrates that $d$ is very much comparable to particle diameter $D$. Adding the Debye length to the particle size makes this correspondence even better. Our interpretation of this glassy state at high concentrations is a jammed state which is appearing at much 
lower volume fractions compared to spheres due to anisotropic shape of disks and their large excluded volume effects. Interestingly Fig. 9 correctly pinpoints the onset of deviations from glassy behavior $(C \approx 2 \mathrm{wt} \%)$.

Recently Ruzicka et al. [8] assigned group $B$ as attractive glass. With this assignment, the transition from gel to glass can be explained simply by that the increase in the volume fraction of the particles leads to the decrease in the void size in gel and eventually the void size decreases nearly to the particle size (attractive glass). Then, however, this scenario can explain neither the absence of the slowing down of the single-particle diffusion for group $B$ nor the absence of the increase in the scattering intensity since attractive glass should accompany the finite-time bond formation between particles and slight increase in the scattering intensity. From this consideration, we suggest that the scenario that group $B$ is a repulsive glass is more plausible.

The assignments of groups $A$ and $C$ are much more subtle. In group $A$ samples (low concentrations, no or little added salt), the aging behavior is distinctly different from that of group $B$. A dramatic decrease of short-time diffusion and remarkable increase of scattered intensity with waiting time is observed. The final structure in such samples is very heterogenous, suggesting the formation of a structure in the form of cluster or gel network. These samples are also characterized by a nonergodicity parameter which reaches the value 1 roughly at a time equal to 2 times the ergodicity breaking time. All these features are consistent with the formation of a gel of Laponite particles, due to attractions between the particles [7]. The assignment of group $A$ as gel has been made by Jabbari-Farouji et al. [7] and more recently also by Ruzicka et al. [8] As shown in Fig. 9, it is obvious that in this range the repulsive interactions are not enough to stabilize a repulsive glass. Nevertheless, it is not so clear why a gel formed by attractive interactions appears in the region of weaker screening of repulsive interactions than the glass. The anisotropic shape of Laponite together with its rim of opposite charges (or neutral) may result in the aggregation in the concentration range. The formation of gel in such a very dilute regime may be a consequence of the competing attractive and repulsive interactions $[33,34]$. Thus, we conclude that $A$ is indeed a gel.

There is a third group of samples, group $C$ samples (moderate Laponite concentrations and high salt content), which also show a heterogenous structure but their aging behavior shares some of the features of group $B$ (glass) and some of the group $A$ (gel). For example, their scattered intensity increases with waiting time (a characteristic of a gel) while their nonergodicity parameter evolution shows a similar behavior to that of group $B$ (a glass). However unlike group $B$, the nonergodicity parameter of the group $C$ does not saturate at a lower value than 1 , but keeps on increasing to reach the value 1 asymptotically at very large $t_{w}>10 t_{e b}$.

Thus, samples in group $C$ share some of the features of the glass, and some of the gel. This could be due to the fact that particles aggregate to form clusters, the diffusion of which becomes hindered progressively as the clusters grow. It has indeed been proposed that such a "cluster glass" exists $[1,22,35-37]$, for which the size of clusters grows in time; this in turn makes that their diffusion significantly slows down. Together with the small amplitude of the motion, the diffusion mode inside a cage may become more and more difficult to observe. This may explain why the nonergodicity parameter reaches 1 at very late waiting times. In suspensions of a similar type of clay (monmorinite), Schurtenberger and his co-workers [1] found cluster fluids in the corresponding dilute region of the phase diagram.

Our experiments show that particles diffusion is suppressed in both group $A$ and $C$ at late times. However, we may need to take special care when interpreting the information of Fig. 4. When we add salt, it is expected that even at $t_{w}=0$ (in our definition) some clusters may have already formed. For example, the scattering intensity at $90^{\circ}$ increases more steeply for group $A$ than for group $C$, but the final scattering intensity is lower for group $A$ than for $C$ [see Fig. $3(\mathrm{~b})]$. This means that at $t_{w}=0$ the samples are more heterogeneous for group $C$ than for $A$. This may be natural on noting that the attractive interaction inducing aggregation is stronger for group $C$ than for $A$. On the other hand, $C$ has characters of both glass and gel, where the slowing down of the dynamics is both due to aggregation (as in a gel) and steric hindrance (as in a glass). The coexistence of these two characters may be quite naturally explained by phaseseparation-induced dynamic arrest: Phase separation leads to a dense region of Laponite, whose composition is high enough for the formation of attractive glass. Such a scenario was proposed for suspensions of uncharged colloids [38]. The strong heterogeneity of the state $C$ is also compatible with this scenario. So we assign the state $C$ as attractive glass formed by phase separation (arrested phase separation due to the formation of attractive glass).

Phase separation requires the presence of attractive interactions. At this moment the nature of the attractive interactions between Laponite particles is unclear. Possible sources are the van der Waals interactions and the attractions between the positive charge on the rim and the negative charge on the surface of Laponite particles. Indeed, recent experiments [39] have shown evidence for a short-range attractive potential in the effective interaction energy.

At this point it is worthwhile comparing the aging features of our attractive glass induced by phase separation with other attraction-driven glassy systems such as the attractive glass formed in hard spheres with added short-ranged attractions [40-42]. In hard sphere systems with added attractions, attractive glasses are formed at moderately high volume fractions of particles and for strong enough attractions. This can be achieved in experiments by adding polymers that cause a depletion interaction $[40,41]$. Light scattering studies in these systems have revealed significant differences between attractive and repulsive glasses in both their static and dynamic properties. Pham et al. [40] showed that upon increasing the attraction strength, entering the attractive glass region (for a fixed volume fraction of colloids), the peak position of the structure factor shifts to a higher $q$ value and its height slightly decreases. This shows that average interparticle distance is decreased upon increasing the attractions and particles bond in clusters, implying that the average number of nearest neighbors should decrease (leading to the decrease in peak height), and holes open up which render the structure more heterogenous on a length scale of a few particles. The 
increased heterogeneity is reflected in a slight increase of the structure factor at low $q$ values. A similar trend is observed in our data upon increasing the salt concentration that screens the repulsions; this should be equivalent to increasing the attractions in the colloid-polymer system.

The differences in dynamics of attractive and repulsive glasses is most evident in the short-time relaxations [40,42]. The short-time dynamics of particles progressively departs from free diffusion upon increasing the attraction. In fact, for attractive glasses the particles are confined so tightly by attractive potential wells that short-time diffusion drops dramatically compared to the repulsive glass at the same particle concentration [40]. This is consistent with our attractive glass samples for which a decrease of the short-time diffusion is observed (Fig. 4). Thus, our attractive glass shares some important similarities with attractive glasses in other systems.

\section{CONCLUSION}

To summarize, we report that there are at least three distinct types of dynamically arrested states in Laponite suspensions. We specify the aging process towards these nonergodic states in detail, using both static and dynamic light scattering. Our data indicate that the competition between short-range (van der Waals) attractions and long-range (electrostatic) repulsions in anisotropic Laponite particles leads to a rich nonergodic state diagram and corresponding aging behavior. On the basis of our data on the static structure factor and the dynamics of the aging, in conjunction with other observations in the literature, we propose that the three observed distinctly different arrested states should be identified as gel $(A)$, repulsive glass $(B)$, and attractive glass $(C)$.

The gel state is formed at low clay concentrations and low amounts of added salt. It has a spatially heterogenous structure as evidenced by our static light scattering measurements. In this case the aggregation of particles either in the form of a networklike structure or clusters is responsible for the aging process. The main characteristics of aging in a gel are dramatic slowing down of translational diffusion and a fast growth of nonergodicity parameter to a fully nonergodic state specified by nonergodicity parameter of value 1 .

The glassy state forms in relatively high concentrations of Laponite and low amounts of added salt. Here, the aging dynamics of a glass has its origin in the cage-diffusion process: For short times or small displacements 'normal' Brownian motion is observed; however, for larger times or excursions, the particles are confined in effective cages formed by their neighbors. This becomes more and more difficult as time goes on, due to the fact that the system finds deeper and deeper free energy minima during the aging process. On the other hand, even for late times the particles maintain their free rattling in the cage, as evidenced by a waiting-time independent short-time diffusion and nonergodicity parameter different from 1 even at the latest stages of aging.

Our study also suggests that a third nonergodic state exists in Laponite suspensions, which we call attractive glass. This state is formed when moderately high amounts of salt share some features of a glass and some of the gel. It has a heterogenous spatial structure similar to a gel while its dynamics is something between that of a gel and a glass.

It is interesting at this point to discuss the relation of our light scattering measurements on these nonergodic states with their rheological properties. Most interesting of course is to see whether a difference between the two glassy states, attractive $(B)$ and repulsive $(C)$ glass, can be found. As described in detail in Ref. [27], we performed local microrheology (MR) experiments on samples belonging to groups $B$ and $C$. In this technique, one looks at the (Brownian) motion of a probe particle, from which the viscoelastic properties of the surrounding medium can be inferred. It was found that that although the complex shear modulus shows a very similar frequency dependence for both types of samples, the local MR measurements reveal the differences in the structure. Local shear moduli obtained from samples of group $B$ are independent of position in the sample while for a sample in group $C$, a significant heterogeneity in the sample develops as the sample ages. Therefore, the shear moduli differ from one position to another in the sample. This provides one more piece of evidence for the classification proposed here. Indeed in [27] we only distinguished between homogeneous samples we called glassy, and heterogeneous samples we called gels. The current paper shows that in fact the heterogeneous samples can be either gels or attractive glasses; this distinction was not made in [27].

\section{ACKNOWLEDGMENTS}

The research has been supported by the Foundation for Fundamental Research on Matter (FOM), which is financially supported by Netherlands Organization for Scientific Research (NWO). LPS de l'ENS is UMR8550 of the CNRS, associated with the universities Paris 6 and 7. H.T. acknowledges a partial support from a grant-in-aid from the Ministry of Education, Culture, Sports, Science and Technology of Japan.
[1] A. Shalkevich, A. Stradner, S. Kumar Bhat, F. Muller, and P. Schurtenberger, Langmuir 23, 3570 (2007).

[2] A. Mourchid, A. Delville, and P. Levitz, Faraday Discuss. 101, 275 (1995); A. Mourchid, A. Delville, J. Lambard, E. Lecolier, and P. Levitz, Langmuir 11, 1942 (1995). A. Mourchid, E. Lecolier, H. V. Damme, and P. Levitz, ibid. 14, 4718 (1998).
[3] D. Bonn, H. Kellay, H. Tanaka, G. H. Wegdam, and J. Meunier, Langmuir 15, 7534 (1999); D. Bonn, H. Tanaka, H. Kellay, G. H. Wegdam, and J. Meunier, Europhys. Lett. 45, 52 (1998).

[4] P. Mongondry, J. F. Tassin, and T. Nicolai, J. Colloid Interface Sci. 283, 397 (2005). 
[5] H. Tanaka, J. Meunier, and D. Bonn, Phys. Rev. E 69, 031404 (2004).

[6] H. Tanaka, S. Jabbari-Farouji, J. Meunier, and D. Bonn, Phys. Rev. E 71, 021402 (2005).

[7] S. Jabbari-Farouji, G. H. Wegdam, and D. Bonn, Phys. Rev. Lett. 99, 065701 (2007).

[8] B. Ruzicka, L. Zulian, R. Angelini, M. Sztucki, A. Moussaid, and G. Ruocco, Phys. Rev. E 77, 020402(R) (2008).

[9] D. W. Thompson and J. T. Butterworth, J. Colloid Interface Sci. 151, 236 (1992); E. Balnois, S. Durand-Vidal, and P. Levitz, Langmuir 19, 6633 (2003).

[10] N. Willenbacher, J. Colloid Interface Sci. 182, 501 (1996).

[11] M. Kroon, G. H. Wegdam, and R. Sprik, Phys. Rev. E 54, 6541 (1996).

[12] F. Pignon, A. Magnin, J. M. Piau, B. Cabane, P. Lindner, and O. Diat, Phys. Rev. E 56, 3281 (1997).

[13] P. Levitz, E. Lecolier, A. Mourchid, A. Delville, and S. Lyonnard, Europhys. Lett. 49, 672 (2000).

[14] B. Abou, D. Bonn, and J. Meunier, Phys. Rev. E 64, 021510 (2001).

[15] D. Bonn, P. Coussot, H. T. Huynh, F. Bertrand, and G. Debrégeas, Europhys. Lett. 59, 786 (2002).

[16] A. Knaebel, M. Bellour, J. P. Munch, V. Viasnoff, F. Lequeux, and J. L. Harden, Europhys. Lett. 52, 73 (2000).

[17] M. Bellour, A. Knaebel, J. L. Harden, F. Lequeux, and J.-P. Munch, Phys. Rev. E 67, 031405 (2003); S. Kaloun, R. Skouri, M. Skouri, J. P. Munch, and F. Schosseler, ibid. 72, 011403 (2005).

[18] T. Nicolai and S. Cocard, Langmuir 16, 8189 (2000).

[19] T. Nicolai and S. Cocard, J. Colloid Interface Sci. 244, 51 (2001).

[20] T. Nicolai and S. Cocard, Eur. Phys. J. E 5, 221 (2001).

[21] S. Cocard, J. F. Tassin, and T. Nicolai, J. Rheol. 44, 585 (2000).

[22] B. Ruzicka, L. Zulian, and G. Ruocco, Phys. Rev. Lett. 93, 258301 (2004).

[23] B. Ruzicka, L. Zulian, and G. Ruocco, Langmuir 22, 1106 (2006); B. Ruzicka, L. Zulian, and G. Ruocco, Philos. Mag. 87, 449 (2007).

[24] S. Jabbari-Farouji, E. Eiser, G. H. Wegdam, and D. Bonn, J. Phys.: Condens. Matter 16, L471 (2004).

[25] R. Bandyopadhyay, D. Liang, H. Yardimci, M. A. Borthwick,
S. G. J. Mochrie, J. L. Harden, and R. L. Leheny, Phys. Rev. Lett. 93, 228302 (2004).

[26] S. Jabbari-Farouji, Ph.D. thesis, University of Amsterdam, 2007.

[27] S. Jabbari-Farouji, M. Atakhorrami, D. Mizuno, E. Eiser, G. H. Wegdam, F. C. MacKintosh, D. Bonn, and C. F. Schmidt, Phys. Rev. E 78, 061402 (2008).

[28] Y. M. Joshi, J. Chem. Phys. 127, 081102 (2007).

[29] B. J. Berne and R. Pecora, Dynamic Light Scattering (Dover, New York, 2000).

[30] P. N. Pusey and W. van Megen, Phys. Rev. Lett. 59, 2083 (1987); W. van Megen, S. M. Underwood, and P. N. Pusey, ibid. 67, 1586 (1991).

[31] CRC Handbook of Chemistry and Physics, edited by R. C. Weast, 63rd ed. (CRC Press, Boca Raton, 1982).

[32] S. Mossa, C. De Michele, and F. Sciortino, J. Chem. Phys. 126, 014905 (2007).

[33] A. Coniglio, L. de Arcangelis, A. de Candia, E. Del Gado, A. Fierro, and N. Sator, J. Phys.: Condens. Matter 18, S2383 (2006).

[34] D. A. Weitz, J. S. Huang, M. Y. Lin, and J. Sung, Phys. Rev. Lett. 54, 1416 (1985); M. Carpineti and M. Giglio, ibid. 68, 3327 (1992); L. Cipelletti, S. Manley, R. C. Ball, and D. A. Weitz, ibid. 84, 2275 (2000).

[35] P. N. Segrè, V. Prasad, A. B. Schofield, and D. A. Weitz, Phys. Rev. Lett. 86, 6042 (2001).

[36] F. Sciortino, S. Mossa, E. Zaccarelli, and P. Tartaglia, Phys. Rev. Lett. 93, 055701 (2004).

[37] K. Kroy, M. E. Cates, and W. C. K. Poon, Phys. Rev. Lett. 92, 148302 (2004).

[38] S. Manley, H. M. Wyss, K. Miyazaki, J. C. Conrad, V. Trappe, L. J. Kaufman, D. R. Reichman, and D. A. Weitz, Phys. Rev. Lett. 95, 238302 (2005).

[39] L. Li, L. Harnau, S. Rosenfeldt, and M. Ballauff, Phys. Rev. E 72, 051504 (2005).

[40] K. N. Pham, S. U. Egelhaaf, P. N. Pusey, and W. C. K. Poon, Phys. Rev. E 69, 011503 (2004).

[41] T. Eckert and E. Bartsch, Phys. Rev. Lett. 89, 125701 (2002); S. H. Chen, W. R. Chen, and F. Mallamace, Science 300, 619 (2003).

[42] A. M. Puertas, M. Fuchs, and M. E. Cates, Phys. Rev. E 75, 031401 (2007). 